\title{
ANORMALIDADES FOCAIS NO PEQUENO MAL EPILÉPTICO
}

\author{
Jose Geraldo Camargo Lima* \\ Rosa HeLENA LONGo* \\ Helio Britto Di Migueli **
}

A epilepsia tipo Pequeno Mal (P.M.) que, na grande maioria dos casos, é a exteriorização clínica de uma disritmia centrencefálica primária, pode ser a tradução sindrômica de uma atividade irritativa focal 1, 8, 10, 11, 12, 13, 20, 21, 22, 23, 24. Esse tipo de crise chamada P.M. focal, com sua disritmia centrencefálica secundária a uma lesão focal veio reforçar a idéia de que fatores orgânicos adquiridos possam estar em causa como sua etiologia. A sua incidência, o seu aspecto clínico e eletrencefalográfico são de importância para o neurologista.

\section{MATERIAL, METODO E RESULTADOS}

O material é constituído por 31 pacientes com crises P.M. cujos eletrencefalogramas mostraram disritmia centrencefálica secundária a atividade irritativa focal. Esse tipo de crise apareceu em nosso material de "disritmias centrencefálicas" (115 pacientes) com uma freqüência de $27 \%$. Dos 31 pacientes, 17 eram do sexo feminino e 14 do masculino; suas idades variaram entre 4 e 14 anos e o tempo de duração da doença entre 2 e 10 anos. Os antecedentes pessoais puderam ser analizados em 21 casos e se mostraram positivos em 7 (33\%) e os antecedentes familiares analizados em 17 casos se revelaram positivos em $3(17 \%)$. Os tipos clinicos das crises estão assinalados na tabela 1 e a topografia da atividade irritativa focal está evidenciada na tabela 2.

Tipos de crise

N.० de casos

\begin{tabular}{lc}
\hline \hline P.M. ausência & 23 \\
P.M. mioclônico & \multicolumn{2}{c}{} \\
P.M. acinético & 4 \\
\hline Tabela 1 - Tipo clínico de crise P.M. \\
$\begin{array}{l}\text { apresentado pelos } 31 \quad p a- \\
\text { cientes. }\end{array}$
\end{tabular}

Topografia N.o de casos

\begin{tabular}{lr}
\hline Frontal D. & 3 \\
Temporal D. & 1 \\
Temporal E. & 2 \\
Temporo-occipital D. & 3 \\
Mesial & 1 \\
Profunda & 21 \\
\hline
\end{tabular}

Tabela 2 - Anormalidades eletrencefalográficas focais apresentadas pelos 31 pacientes.

Trabalho do Departamento de Neurologia e Neurocirurgia da Escola Paulista de Medicina, apresentado no V.o Congresso Brasileiro de Neurologia e III Congresso Brasileiro de Eletrencefalografia e Neurofisiologia Clinica (São Paulo 12-15 julho 72): * Professor Adjunto; ** Assistente. 
C O M E N TA R I O S

De incidência variável entre as crises P.M. (3 a 20\% $)^{2}, 3,7,9,10,14,15,18,20$, o P.M. focal foi assinalado em $3 \%$ dos casos de epilepsia de um modo geral 18. Entre os nossos pacientes com "disritmia centrencefálica" o encontramos com uma freqüência de $27 \%$. Como foi assinalado por Jasper ${ }^{11}$, nem sempre a manifestação clínica é P.M. típico (ausência) pois outros tipos de crise podem ocorrer. Dos nossos pacientes, 4 apresentaram P.M. mioclônico e 4 P.M. acinético. A analize da topografia da atividade irritativa mostrou que em 10 casos $(8 \%)$ era cortical e, em 21 casos (19\%), era profunda, o que está de acordo com as idéias de Gastaut, citado por Loiseau e Cohadon ${ }^{14}$. Como assinalam Calderon e Pall ${ }^{2}$, Nuffield ${ }^{18}$ e Niedermeyer ${ }^{17}$, a atividade irritativa cortical predominou em áreas temporais; Loiseau e Cohadon ${ }^{14}$ assinalam predominância em áreas frontais. Os antecedentes pessoais analizados no P.M. de um modo geral, mostram ser de valor em uma freqüência extremamente variável: 1,8\% para Gibbs e Gibbs ${ }^{7}$ e $30 \%$ para Miribel ${ }^{16}$. Dalby ${ }^{4}$ assinala que no P.M. focal os antecedentes pessoais positivos são extremamente freqüentes $(58 \%)$. No nosso material esse fato foi assinalado em $33 \%$ dos casos. Consideramos esse dado de importância pois vem mostrar que o fator lesional adquirido tem realmente valor significativo na gênese desses quadros. Entre nós Pupo ${ }^{21}$ ressaltou bem esse ponto ao apresentar o caso de dois gêmeos univitelinos com quadro de P.M. típico, com eletrencefalograma nitidamente focal e antecedentes pessoais de sarampo.

\section{R E S U M O}

Foram estudados 31 pacientes com crises clínicas tipo P.M. cujos eletrencefalogramas revelaram disritmia centrencefálica secundária a atividade irritativa focal. A sua freqüência entre as disritmias centrencefálicas foi de $27 \%$ (19\% secundárias a foco de localização profunda e $8 \%$ secundárias a foco de localização cortical). O tipo clínico das crises foi analizado, sendo ressaltado o fato de que 8 pacientes não apresentaram crise tipo P.M. puro (ausência) : 4 apresentaram P.M. mioclônico e 4 P.M. acinético. A alta incidência de antecedentes pessoais de valor (33\%) foi enfatizada.

\section{S U M M A R Y}

\section{EEG focal abnormalities in P.M. epilepsy}

Thirty one patients with P.M. epileptic crisis whose EEG showed centrencephalic disrythmia secondary to focal irritating activity where thoroughly observed. $19 \%$ of the cases showed centroencephalic disrythmia secondary to deeply situated focus and $8 \%$ to focus of cortical situation; in these cases the temporal projection was predominant. The clinical type of the 
crisis was analysed with emphasis on the fact that 8 patients did not show a "sheer" P.M. type crisis (absenca) : 4 showed mioclonic P.M. and 4 akynetic P.M. The high incidency of personal antecedents $(33 \%)$ is emphasized.

$R$ E F E R E N I A S

1. BICKFORD, R. G. - The application of depth electrography in some varieties of epilepsy. Electroenceph. Clin. Neurophysiol. 8:526, 1956.

2. CALDERON, A. \& PALL, G. - Focal changes and EEG in P.M. epilepsy. Electroenceph. Clin. Neurophysiol. 9:350, 1957.

3. CLARK, E. E. \& KNOTT, J. R. - Paroxysmal wave and spike activity and diagnostic subclassification. Electroenceph. Clin. Neurophysiol. 7:161, 1955.

4. DALBY, M. A. - Epilepsy and 3 per second spike and wave rhythms. A clinical EEG and prognostic analysis of 346 patients. Acta Neurol. Scand. Supl. 40:1, 1969.

5. GIBBERD, F. B. - The prognosis of P.M.. Brain. 89:531, 1966.

6. GIBBERD, F. B. - The clinical features of P.M. Acta Neurol. Scand. 42: 176-90, 1966.

7. GIBBS, F. A. \& GIBBS, E. L. - Atlas of Electroencephalography. AddisonWesley Press. Inc., Massachusetts, 1957.

8. GLASER, H. G. - The concept of centrencephalic epilepsy and the spike and wave EEG. Electroenceph. Clin. Neurophysiol. 9:172, 1957.

9. HOLLOWACH, J.; THURSTON, D. L. \& O'LEARY, J. L. - P.M. epilepsy. Pediatries 30:893, 1962.

10. JASPER, H. \& KERSHMAN, J. - Electroencephalographic classification of the epilepsies. Arch. Neurol. Psychiat. (Chicago) 45:903, 1941.

11. JASPER, H. - Reflections on the spike and wave complex in cortical and centrencephalic seisures. Electroenceph. Clin. Neurophysiol. 9:379, 1957.

12. JUNG, R. - Zur Klinik Electrophysiologie des P.M., An. IV Congrés Internat. d'Electroencephalographie et Neurophysiologie Clinique (Bruxelles) 296, 1957.

13. LENNO, M. A. \& ROBINSON, R. - Cingulate-cerebellar mechanisms in the physiological pathogenesis of epilepsy. Electroenceph. Clin. Neurophysiol. $3: 197,1951$.

14. LOISEAU, P. \& COHADON, F. - Le Petit Mal et ses Frontières. Masson \& Cie., Paris, 1970.

15. LORENTZ de HAAS, A. M. \& STOEL, L. M. K. - Experiences with a ethylmethyl succinimide in the treatmant of epilepsy. Epilepsia (Amesterdam) 1: $501,1960$.

16. MIRIBEL, J. - Aspects Cliniques du Petit Mal Epileptique chez l'Enfant. Doin Ed., Paris, 1963.

17. NIEDERMEYER, E. - The occurense of centrencephalic and focal seizure patterns in the same patient. Electroenceph. Clin. Neurophysiol. 23:87, 1967.

18. NUFFIELD, E. J. A. - Electro-clinical correlations in childhood epilepsy. Épilepsia (Amsterdam) 2:178, 1961.

19. O'BRIEN, J. L.; GOLDENSOHN, E. S. \& HOEFER, P. F. - EEG abnormalities in addition to bilaterally synchronous 3 per second spike and wave activity in P.M. Electroenceph. Clin. Neurophysiol. 11:747, 1959.

20. PUPO, P. P.; PAZZANESE, O. \& LONGO, R. H. - Epilepsia "Petit Mal". Arq. Neuro-Psiquiat. (São Paulo) 19:101, 1961. 
334 ARQ. NEURO-PSIQUIAT. (SÄO PAULO) VOL. 30, N.o 4, DEZEMBRO, 1972

21. PUPO, P. P. - Convulsões na infância: possiveis fatores predisponentes. Fatores genéticos e fatores adquiridos por ocasiāo do parto. Rev. paul. Med. (São Paulo) 61:199, 1962.

22. PASSqUANT, P. \& CADILHAC, J. - EEG and clinical study of epilepsy during maturation in man. Epilepsy (Amsterdam) 3:14, 1962.

23. RICCI, G. \& VIZIOLI, R. - Complessi punta-onda bilaterali e sincroni e focali corticali. Riv. Ital. di Neurol. 24:808, 1954.

24. TUKEL, K. \& JASPER, H. - The electroencephalogram in parassagital lesions. Electroenceph. Clin. Neurophysiol. 4:481, 1952.

Departamento de Neurologia e Neurocirurgia - Escola Paulista de Medicina - Caixa Postal 5496 - 01000 São Paulo, SP - Brasil. 